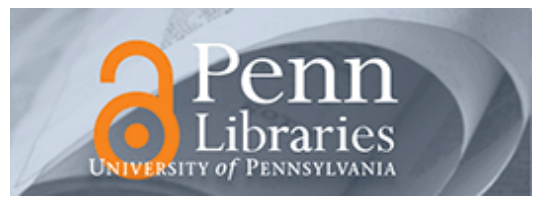

University of Pennsylvania ScholarlyCommons

$11-5-2018$

\title{
Reactive Velocity Control Reduces Energetic Cost of Jumping with a Virtual Leg Spring on Simulated Granular Media
}

Sonia F. Roberts

University of Pennsylvania, soro@seas.upenn.edu

Daniel E. Koditschek

Electrical and Systems Engineering, University of Pennsylvania

Follow this and additional works at: https://repository.upenn.edu/ese_papers

Part of the Power and Energy Commons, and the Systems Engineering Commons

\section{Recommended Citation}

Sonia F. Roberts and Daniel E. Koditschek, "Reactive Velocity Control Reduces Energetic Cost of Jumping with a Virtual Leg Spring on Simulated Granular Media", Proceedings of the 2018 IEEE International Conference on Robotics and Biomimetics . November 2018. 


\title{
Reactive Velocity Control Reduces Energetic Cost of Jumping with a Virtual Leg Spring on Simulated Granular Media
}

\author{
Abstract \\ Robots capable of dynamic locomotion behaviors and high-bandwidth sensing with their limbs have a \\ high cost of transport, especially when locomoting over highly dissipative substrates such as sand. We \\ formulate the problem of reducing the energetic cost of locomotion by a Minitaur robot on sand, reacting \\ to robot state variables in the inertial world frame without modeling the ground online. Using a bulk- \\ behavior model of high-velocity intrusions into dry granular media, we simulated single jumps by a one- \\ legged hopper using a Raibert-style compression-extension virtual leg spring. We compose this controller \\ with a controller that added damping to the leg spring in proportion to the intrusion velocity of the robot's \\ foot into the simulated sand while the robot is pushing off in the second half of stance. This has the \\ effect of both reducing the torque exerted by the motors because the added virtual "active damping" force \\ acts in opposition to the virtual leg spring force, and reducing the transfer of energy from the robot to the \\ sand by slowing the intrusion velocity of the foot. Varying the simulated robot's initial conditions and the \\ simulated ground parameters, we gained a consistent $20 \%$ energy savings by adding active damping with \\ no cost in apex height. \\ For more information, see the Kod*lab website: kodlab.seas.upenn.edu \\ Keywords \\ legged locomotion, granular media, energy management \\ Disciplines \\ Electrical and Computer Engineering | Engineering | Power and Energy | Systems Engineering
}

This conference paper is available at ScholarlyCommons: https://repository.upenn.edu/ese_papers/841 


\title{
Reactive velocity control reduces energetic cost of jumping with a virtual leg spring on simulated granular media
}

\author{
Sonia Roberts ${ }^{1}$ and Daniel E. Koditschek ${ }^{1}$
}

\begin{abstract}
Robots capable of dynamic locomotion behaviors and high-bandwidth sensing with their limbs have a high cost of transport, especially when locomoting over highly dissipative substrates such as sand. We formulate the problem of reducing the energetic cost of locomotion by a Minitaur robot on sand, reacting to robot state variables in the inertial world frame without modeling the ground online. Using a bulk-behavior model of high-velocity intrusions into dry granular media, we simulated single jumps by a one-legged hopper using a Raibertstyle compression-extension virtual leg spring. We compose this controller with a controller that added damping to the leg spring in proportion to the intrusion velocity of the robot's foot into the simulated sand while the robot is pushing off in the second half of stance. This has the effect of both reducing the torque exerted by the motors because the added virtual "active damping" force acts in opposition to the virtual leg spring force, and reducing the transfer of energy from the robot to the sand by slowing the intrusion velocity of the foot. Varying the simulated robot's initial conditions and the simulated ground parameters, we gained a consistent $20 \%$ energy savings by adding active damping with no cost in apex height.
\end{abstract}

\section{INTRODUCTION}

\section{A. The Ghost Minitaur robot can be used to measure ground erodibility but cannot locomote effectively on sand}

To collect data relevant to geoscientific experiments in hostile environments such as deserts, we employ a heterogeneous team of robots, including both a "pack mule" X-RHex [1] robot carrying large payload of sensors [2], [3] and a fast, dynamic Minitaur [4] robot with legs that can be used as force sensors to quickly measure ground properties relevant to erodibility over a large area [5]. Here, we target the Ghost Minitaur robot, ${ }^{1}$ a quadrupedal robot with direct-drive legs that can be programmed for purposes of locomotion using the composition of virtual damped springs [6]. The proprioceptive transparency [7] of the direct-drive legs allows their use as force sensors to study the erodibility of soils under mechanical shear stress, a measure of interest for desert research [5]. However, due to the inefficiency of motors in the high-torque, low-velocity regime required for direct-drive legged robot locomotion, Minitaur is currently unable to run in the highly taxing desert environment. Forcesensing experiments in the field are currently conducted with a single direct-drive Minitaur leg mounted on the back of a heavily geared X-RHex, leaving little room for additional sensors.

\footnotetext{
${ }^{1}$ Electrical and Systems Engineering, University of Pennsylvania, Philadelphia, PA. Contact: soro at seas dot upenn dot edu

${ }^{1}$ Ghost Robotics, 3401 Grays Ferry Ave, Philadelphia, PA 19146 http://www.ghostrobotics.io
}

In previous experiments using robots to collect data in deserts, X-RHex was made desert-ready with an increase to its gearbox ratio, wider feet, and stiffer legs [5], but these options are undesirable choices for Minitaur. The addition of a gearbox would reduce actuator transparency [7] and make the robot unable to act as a force sensor; the foot size can be increased by about a factor of two, but too large a foot and the inertia will again render the force sensor useless; and stiffening the legs comes at a high cost from the battery, since the spring force is virtual rather than mechanical. The increase in gearbox ratio has also slowed X-RHex down significantly: whereas a standard X-RHex can keep up with a jogging or running human on rugged terrain [8], the highly geared desert version has a top speed at best closer to human walking speed [5].

\section{B. Previous research has produced bulk-behavior models with predictive power for locomotion on granular media}

Bulk-behavior models of granular media developed for the purposes of modeling animal and robot locomotion characterize ground reaction forces in response to intrusion by a limb [9], [10]. In general, bulk-behavior models of the plastic flows of granular media are accurate when the size of the intruder far exceeds the size of the grains [11], and the results scale well with different sizes and masses of intruders [12]. Bulk-behavior models can now predict the terrain response with sufficient accuracy to allow optimal control methods to generate robot motion trajectories that result in jumps to a desired height [13], [14]. Although powerful, results using optimal control have limited applicability to robot locomotion on real deserts, as they assume granular media preparations that are homogeneous within and between steps - an assumption which cannot be made for locomotion in real deserts with unknown ground properties [5], [3], [2] - and experiments in the laboratory are not much affected by the dissipation of electrical energy to heat, which is a very real concern for a robot running long distances in a desert.

We use the same added-mass bulk-behavior model [9] that was validated in the highly dynamic regime by its use in optimal control experiments. Three forces comprise the bulkbehavior model: A stiffness function only of depth, $k_{g}(x)$; an inertial drag energy dissipation term which is a function of depth and velocity, $d_{g}(x) \dot{x}^{2}$; and an added mass term, $m_{a}(x) \ddot{x}$, which describes the mass of the grains recruited to a stagnant "cone"-shaped clump of grains accelerated underneath the intruding robot foot. The full model [9] is 
therefore:

$$
F_{g}=k_{g}(x)+d_{g}(x) \dot{x}^{2}+m_{a}(x) \ddot{x} .
$$

We describe the three terms in detail.

1) The depth-dependent stiffness function, $k_{g}(x)$ : The depth-dependent force described in the added-mass model uses Resistive Force Theory (RFT) [10], a bulk-behavior model describing the hydrostatic-like forces of granular media in response to intrusion. The force response is transiently nonlinear when depths are very low, which is attributed to the growth of the cone of grains accelerating along with the intruding foot. Once the cone is fully formed, the force response is linear in depth, with $k_{g}(x)=k_{g c} \cdot x$ for some constant $k_{g c}$. The force response scales with the surface area of the foot, and thus the projected area of the cone of grains moving under the foot, with a larger foot surface area for a given animal or robot significantly improving locomotion capability [15]. RFT models the force response at arbitrary depths and angles using experimentally determined or measured parameters about the individual grains. These hydrostatic-like forces during vertical intrusion have recently been modeled in a universally scalable form using only the internal friction angle, that is, the angle relative to the normal force obtained at the point of failure of the granular media in response to a shearing stress [16].

2) The depth-dependent inertial drag term, $d_{g}(x) \dot{x}^{2}$ :

This term describes the hydrodynamic-like forces arising from the inertia of the grains accelerated underneath the robot's foot and recruited into the cone of added mass. The form of the term arises from the rate at which the mass is added through the recruitment of grains and then the shearing (and continued recruitment and shedding) of grains along the sides of the cone. While the cone is forming, the depth-dependent function describing the rate of recruitment of grains contributes a transient nonlinearity to the $d_{g}(x)$ term. Once the cone is formed, this term is constant in depth and quadratic in velocity. Studies of energy dissipation from high-velocity vertical collisions [17], [18] support the interpretation of this term as a dissipation function.

3) The added mass term, $m_{a}(x)$ : The added mass term describes the mass of the developing cone of grains that are accelerated along with the robot's foot. The development of this cone of grains, and the shearing forces along the cone once it is fully developed, are also modeled in the depthdependent inertial drag energy dissipation term: the transient non-linearity in $d_{g}(x)$ is explained as the growth of the cone, and the linearity thereafter is explained as the recruitment and shedding of grains along the shearing plane of the cone of grains pushed down under the foot.

$C$. We reduce the energetic cost of jumping on granular media without assuming knowledge of its properties, reacting locally to inertial-frame robot state

As our nominal comparison controller for Minitaur running on sand, we use a simple compression-extension spring control law proposed originally by Raibert [19] and used for a suite of behaviors and applications, including recently,
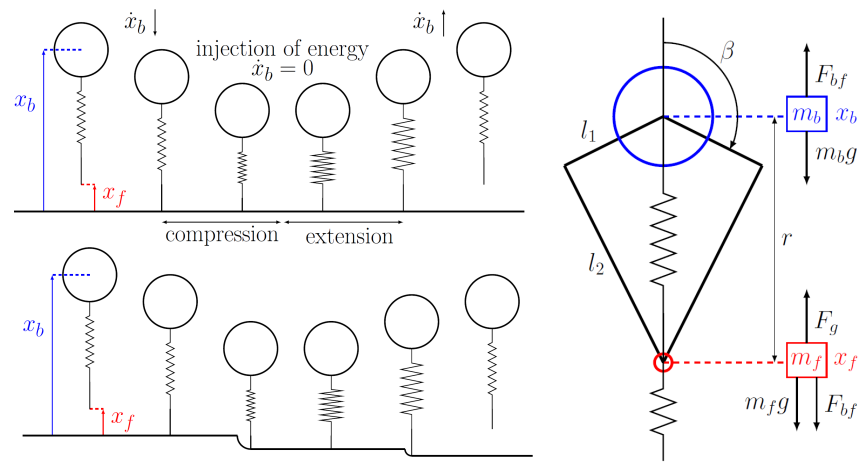

Fig. 1: (Left) A Raibert-style compression-extension spring controller on rigid (top) and compressible ground (bottom). The soft (thin) virtual leg spring compresses easily. When fully compressed, the virtual spring is instantaneously switched to a very stiff (thick) virtual spring. (Right) The kinematic diagram of the simulated onelegged hopper shows the virtual spring created by a simple linkage using two opposing motors, and the forces from the robot's leg, the ground, and the masses of the body and foot during stance.

parallel compositions of behaviors [6], [20], [21]. A soft compliance gain during the first part of stance allows the virtual leg spring to compress. Once the rate of change of the leg length goes to zero, the programmed leg stiffness increases by a large amount instantaneously, injecting a large quantity of potential energy into the virtual spring. This causes the robot to jump. During the switch from soft compression spring to stiff extension spring on sand, in addition to causing the robot's body to accelerate upwards, the forces from the leg spring also push the foot further into the sand. (See Figure 1.)

The energetic cost is considered to be the cost in joules from a battery required to perform a single hop. In simulation experiments, a simple motor model, battery model, and the kinematics of the leg are used to estimate the cost in joules. A hopping robot constrained to a linear rail was used to relate the estimated cost used in simulations to the cost of jumping as measured by the power drawn from a battery.

\section{Methods}

\section{A. Granular media simulation in Matlab}

Computer simulations were performed in Matlab using a discrete-time dynamical system with a small time-step $\left(\mathrm{dt}=1 \times 10^{-6} \mathrm{~s}\right) .^{2}$ The added-mass bulk-behavior model uses measured parameters about the individual grains of the granular media, and has two additional scalars in the dissipation function describing the hydrodynamic-like forces: One

\footnotetext{
${ }^{2}$ In [9], where a Matlab simulation was also used to simulate the bulk-behavior of granular media using this added-mass model, Zeno-like switching between unjammed and jammed granular modes was addressed by switching the simulated granular media from a mode in which compression is allowed to a rigid "jammed" mode by watching the acceleration of the foot. We selected our time-step to be small enough that there was no Zenolike switching in the behavior of the robot, but allowed Zeno-like switching from the granular media. We then recalculated the values of the ground's stiffness, dissipation, and added mass terms afterwards based on the robot foot's simulated behavior, which did not have this switching. This approach allowed the granular media to jam, unjam, and re-jam an arbitrary number of times during stance as the forces from the leg changed.
} 
Bulk-behavior granular media model used in simulation
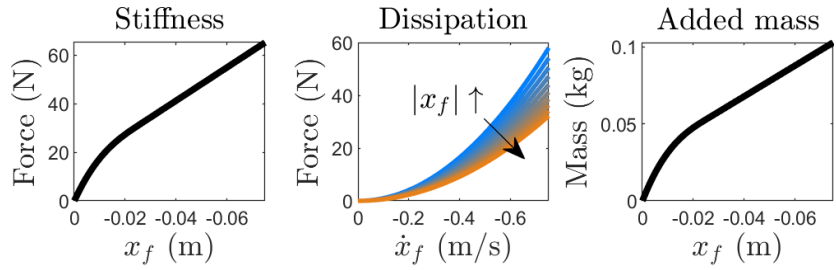

Fig. 2: The stiffness, dissipation, and added mass functions of the simulated granular media. The stiffness function is transiently nonlinear while the cone of grains accelerated with the foot is still forming. The depth-dependent dissipation function is quadratic in velocity, with a higher coefficient while the cone forms. The added mass function is not constant after the cone forms because the cone continues to shed and gain material throughout intrusion.

a linear scaling parameter which was experimentally fitted in [14], and one a constant from integration. The constant from integration arises from the derivation of the added mass term in [9], which was integrated from a change in added mass and reflects the inertia of the grains accelerated along with the intruding foot. To ease comparison with and extension from previous results, for our nominal granular media we used parameters that were measured for poppyseeds [10], and the same linear scaling parameter $\left(b_{g}=17.2\right)$ as in [14]. We set the integration constant $C=1$. As the difference between the contributed and the nominal controllers occurs only after the transient nonlinearities during the formation of the cone of added material in the stiffness, dissipation, and added mass functions of the ground, these details do not greatly affect the results (see Section II-F.2 and Figure 9).

The three component terms $k_{g}\left(x_{f}\right), d_{g}\left(x_{f}\right) \dot{x}^{2}$, and $m_{a}\left(x_{f}\right)$ used to simulate the granular media response to intrusion by the robot's foot are plotted in Figure 2. The ground reaction model exerts no forces when the robot's foot velocity is positive, and all added mass is lost when the foot lifts off. This simulates the plastic deformation of the ground and allow for continued deformation during leg extension with a stiff virtual extension spring following the initial deformation under the soft virtual compression spring.

\section{B. Nonlinear two-spring model of Minitaur locomotion on granular media}

We consider a one-dimensional hopping robot with a body mass of $1.75 \mathrm{~kg}$ and a foot mass of $0.175 \mathrm{~kg}$ (10\% of body mass) constrained to move vertically. The robot is modeled on one quarter of a lightweight, desert-ready version of the Minitaur robot currently under development. Connecting the body and foot masses is a programmable leg spring with rest length $l=0.27 \mathrm{~m}$ which can measure its own length and velocity, with an update loop of $1 \mathrm{kHz}$ (achievable on Minitaur robots [4]) and a stiffness of $k_{l}$. The leg spring has a small linear dissipation coefficient, $d_{l}>0$. To implement the contributed controller, we assume that the robot can sense the depth of its foot in an inertial frame.

A hop has two modes: Stance, during which time the robot's foot is in contact with the ground, and flight, when the robot's body and foot are both aerial. The dynamics of the two modes are as follows:

1) Stance: The accelerations of the body and foot are governed by a two-mass, two-spring nonlinear dynamical system. Letting $x_{b}^{n}, x_{f}^{n}$ be the robot body and foot centers of mass at time $n, k_{l}$ and $d_{l}$ the linear stiffness and damping coefficients of the robot leg, with $g$ the gravitational constant, we can define first the forces of the virtual leg spring,

$$
F_{b f}=k_{l}\left(x_{b}-x_{f}-l\right)+d_{l}\left(\dot{x}_{b}-\dot{x}_{f}\right) .
$$

Adding now $m_{b}, m_{f}^{n}=m_{f}\left(x_{f}^{n}\right)$ the masses of the robot body and foot respectively and $k_{g}^{n}=k_{g}\left(x_{f}^{n}\right), d_{g}^{n}=d_{g}\left(x_{f}^{n}\right)$ and with it, we define the accelerations of body and foot at time $n$ in stance:

$$
\begin{aligned}
\ddot{x}_{b}^{n} & =-\frac{F_{b f}}{m_{b}}-g \\
\ddot{x}_{f}^{n} & =\frac{F_{b f}}{m_{f}^{n}}-\frac{k_{g}^{n}}{m_{f}^{n}} x_{f}^{n}-\operatorname{sign}\left(\dot{x}_{f}^{n}\right) \frac{d_{g}^{n}}{m_{f}^{n}}\left(\dot{x}_{f}^{n}\right)^{2}-g .
\end{aligned}
$$

The switch from stance to flight mode occurs when the following condition is met, for $\delta$ the leg spring compression due to gravity from the body's mass: $k_{l}\left(x_{b}^{n}-x_{f}^{n}-(l-\right.$ $\delta))+d_{l}\left(\dot{x}_{b}^{n}-\dot{x}_{f}^{n}\right)>m_{f}^{n} g$. In flight, the ground stiffness and dissipation functions go to zero, and the added mass is immediately lost.

2) Ballistic flight: Once the foot lifts off from the compressed ground, the virtual leg spring switches to its soft compression gain. The body and foot are considered to be in free flight and their trajectories determined by standard flight dynamics. The transition from flight to stance occurs when the foot touches the ground, $x_{f}=0$, and the body velocity is negative.

C. The contributed "active damping" controller adds damping to the leg spring when the foot continues to compress the ground while pushing off

The nominal compression-extension controller and the contributed active damping controller differ only in the extension portion of stance and only when the velocity of the foot in the inertial world frame is negative. Under these conditions, the active damping controller adds this force:

$$
F_{A D}=-\left(b_{A D} \cdot \dot{x}_{f}\right) \cdot\left(\dot{x}_{b}-\dot{x}_{f}\right), \quad \dot{x}_{f}<0 .
$$

We used $b_{A D}=k_{l}$, which during extension is the stiff gain.

\section{Kinematics and motor model used for energy estimates}

We used the kinematics of the virtual leg spring created by the simple linkage to estimate the motor torques and therefore the current draw from the battery required to exert the forces at the foot in the simulated experiments. The kinematics were simplified by the assumption that the leg and foot are constrained to move vertically. For $l_{1}, l_{2}$ the leg linkage lengths, $r$ the length from the center of mass of the motors to the foot, and $\beta$ the angle of the first link relative to the vertical, the inverse kinematics are $\pi-\beta=\cos ^{-1}\left(\frac{l_{1}^{2}-l_{2}^{2}+r^{2}}{2 l_{1} r}\right)$. The torque for leg spring force $F_{b f}$ is then $\tau=D f \mid{ }_{r} F_{b f}$. (See Figure 1.) To estimate losses, 
we assumed that the leg looked mechanically like a perfect linear spring, calculated the torques at the motors required to produce the forces at the foot, and then calculated the additional energy lost to heat and electromechanical drag in order to achieve those torques.

A simple motor model relating the current draw to the torque consisting of a linear portion with instantaneous saturation at the stall torque was used. For $K_{\tau}$ the motor torque constant, $\tau_{n}$ the torque from the motor at time-step $n$, and $I_{n}$ the estimated current draw at time-step $n$, we have $\tau_{n}=K_{\tau} \cdot I_{n}$. For any torques requested by the simulation above the stall torque for two motors working together to rotate the first leg links, $2 \cdot \tau_{s}$, we provided the stall torque of the leg instead. Losses to heat were calculated as $P_{R_{m}}=I^{2} R_{m}$ and losses to electromechanical drag were calculated as $P_{v}=\frac{K_{\tau} K_{v}}{R_{m}} \dot{\beta}^{2}$, for $K_{v} \frac{V}{\mathrm{rad} / \mathrm{s}}$ the motor velocity constant and $R_{m} \Omega$ the motor resistance. The electrical power converted to mechanical power was calculated as $\dot{\beta} \tau$. The values for $K_{\tau}=0.0955 \frac{\mathrm{Nm}}{\mathrm{A}}$, the stall torque $\tau_{s}=3.5 \mathrm{Nm}$, and motor resistance $R_{m}=0.186 \Omega$ for the T-motor U-8 series motors used on Minitaur were reported in a previous study on actuator sizing [7].

With $T$ the number of time-steps, $I_{n}$ the current draw of the motor, $\beta_{n}$ the motor angle, and $\tau_{n}$ the torque at the motor at time-step $n$, the energetic cost $J$ of a single jump is then:

$$
J=\mathrm{dt} \sum_{n=1}^{T}\left[R_{m} I_{n}^{2}+\dot{\beta}_{n} \tau_{n}+\frac{K_{\tau} K_{v}}{R_{m}} \dot{\beta}_{n}^{2}\right] .
$$

This is the sum of the losses to heat through the resistance of the motor, losses to electromechanical drag, and the mechanical energy converted from electrical energy over each time-step. Direct-drive motors operating at high torques and low velocities are inefficient, and the cost is dominated by the dissipation to heat.

\section{E. Test of simulation by hopping a robot with different extension gains on a force plate}

To validate the energy cost estimates of the simulated robot, we compared simulated costs with energy costs measured from a battery used to power a $2.0 \mathrm{~kg}$ one-legged robot hopping on a Bertec force plate ${ }^{3}$ while constrained to a vertical rail. These experiments serve as a ground truth for the robot model used in simulations. The robot was programmed to jump using the compression-extension controller with four extension virtual stiffness gains, and completed 80 jumps during each experiment. The battery used to power the robot was fully charged before and after each experiment, and the mAh required to fully recharge the battery after the experiment as reported by the ThunderPower charger was recorded as the cost of 80 jumps using that virtual stiffness gain during extension.

To control for variability due to changing motor resistance as the motors warm up, we allowed the robot to jump for approximately 2 minutes before any experimental data was

\footnotetext{
${ }^{3}$ Bertec FP4060-07 force plate, leveled to within 1 degree in either axis of the horizontal plane; data recorded using Bertec Acquire version 4.0.12.411.
}

taken. We also used block experiment ordering to prevent an interaction between time and leg stiffness. For extension gains $k_{l}=\{300,400,500,600\}$, we performed experiments in this order: $\{300,600,500,400\}$. Each experiment began with the robot leg held just above the force plate, such that when it was released the soft virtual leg spring immediately compressed under the weight of the body. When the robot's body velocity reached zero, the robot switched to its stiff extension gain and jumped. Within a few hops (two or three), the robot reached a consistent jumping height in all extension gain conditions. The robot was allowed to jump 80 times and was caught by the experimenter while in flight after the 80th jump. The battery was immediately disconnected to avoid any additional power draw.

In addition to vertical axis force data $(\mathrm{N})$ recorded from the force plate, we collected leg extension position and velocity data as reported by the Ghost SDK. For ground truth, we recorded the height of the robot's body through the SDK using a spring potentiometer, and took record-keeping videos which were not analyzed as data. The force plate data was recorded at $1 \mathrm{kHz}$, and robot data was logged at $333 \mathrm{~Hz}$. The force plate data was lightly smoothed with a low-pass filter to reduce noise (moving average filter, window size of 15 samples $=0.015 \mathrm{~s}$ ). The window size was chosen by plotting the sum of absolute differences between filtered and unfiltered data for a range of window sizes, and picking the window size at which the difference between one filtered force profile and the next becomes (and stays) small.

The force plate and leg extension data were automatically aligned at the leg touchdown for each hop, which was automatically detected in the robot by leg deflection and on the force plate by a large spike in the raw force plate readings (Figure 3). The force plate data, which was zeroed from the mass of the robot resting on the force plate, was used to determine liftoff at the end of stance. For each hop, the leg lengths and ground reaction forces from the force plate were fed directly into the kinematic model and the motor model described in Section II-D. An estimate for the number of joules required to exert the forces at the foot as reported by the force plate at the leg lengths reported by the SDK was calculated. Assuming the leg was moving only vertically, we first estimated $\hat{\beta}$ (see Figure 1) using the reported leg length $r$, and then calculated $\hat{\tau}=D f_{r} F_{f p}$ using the force plate data $F_{f p}$. (In computer simulations, the force exerted by the toe was determined by the leg spring constant and damping coefficient.) $\hat{\beta}$ was used to estimate the losses to electromechanical drag and we used the inverse motor model to calculate the estimated losses to heat $\hat{P}_{R_{m}}$. The motor models used for simulation and for validation experiments differed only in that we did not assume a stall torque in the validation experiments, so that the forces measured by the force plate could supersede the theoretical maximum forces the leg could exert based on the stall torque. This estimated joules $\hat{J}$ (see Eqn 2 ) for each hop was then plotted against the measured average joule cost $\bar{J}$ for a single jump in that condition (Figure 3 ). The measured and estimated joules had a strong correlation that was close 
Estimated and measured joules cost per hop

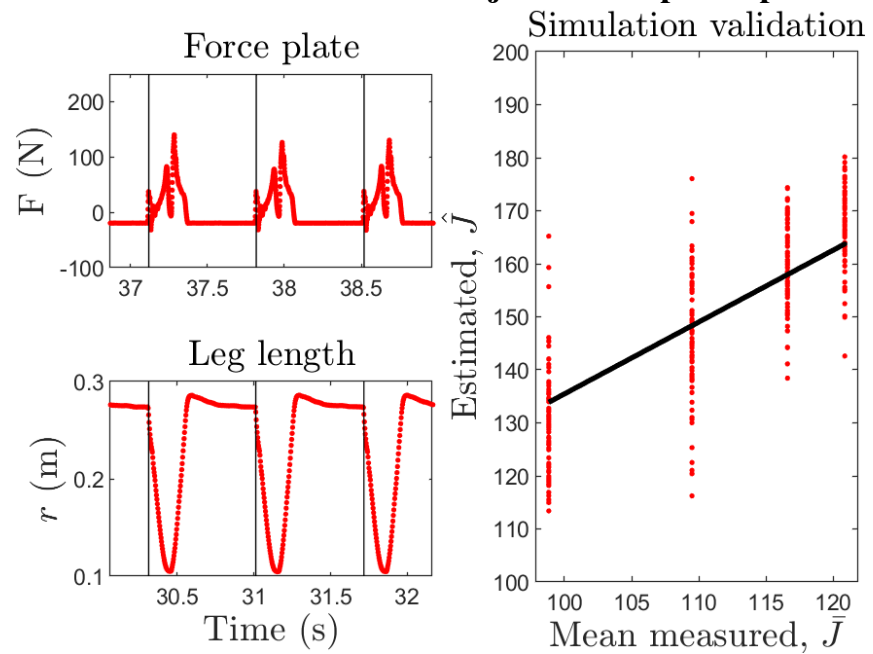

Fig. 3: The force plate data (left, top) and leg position data (left, bottom) were both repeatable between jumps. The force plate readings were zeroed from the weight of the robot. The black vertical bars indicate the automatically detected onset of stance. The mean measured joules per hop $\bar{J}$ was well correlated with the estimate of the joules cost $J$ (Eqn 2; Section II-E) calculated using the forces measured by the force plate and the leg position data (right), $\hat{J}=1.35 \bar{J}$, RMSE/IQR $=0.63$. The variability comes from the force plate data, which is only lightly filtered.

to unity $(\hat{J}=1.35 \bar{J}, \mathrm{RMSE} / \mathrm{IQR}=0.63)$.

F. Computer simulations using the bulk-behavior force model show little effect of ground parameter or initial condition variation on energy savings

We performed computer simulations in Matlab of a onelegged hopper with a virtual leg spring performing a single jump on granular media using the added-mass bulk-behavior model, varying ground parameters and initial conditions.

1) The active damping controller consistently loses less energy than the compression-extension controller under different initial conditions: We varied the initial velocity of the body and foot, assuming that both were starting at the same velocity and with a neutral leg length. Since the virtual spring stiffness gain during the initial compression phase of stance is very anemic, and the foot has only $10 \%$ of the mass of the body, there are only small effects on the estimated energy costs from varying either initial positions or different initial velocities for the body and foot, and the largest effects are seen by varying the amount of kinetic energy that the robot starts with at the beginning of stance. Plotting the energy cost of a jump using the active damping and compressionextension controllers with a variety of initial body velocities (Figure 4), we see a consistent savings of $20 \%$.

2) The active damping controller consistently loses less energy than the compression-extension controller under different ground conditions: In the stiffness function, we varied $\sigma$, the parameter describing the stress in the vertical direction. Once the cone of added material is formed and the stiffness function becomes linear in depth, this parameter is the scalar coefficient determining the stiffness of the ground.

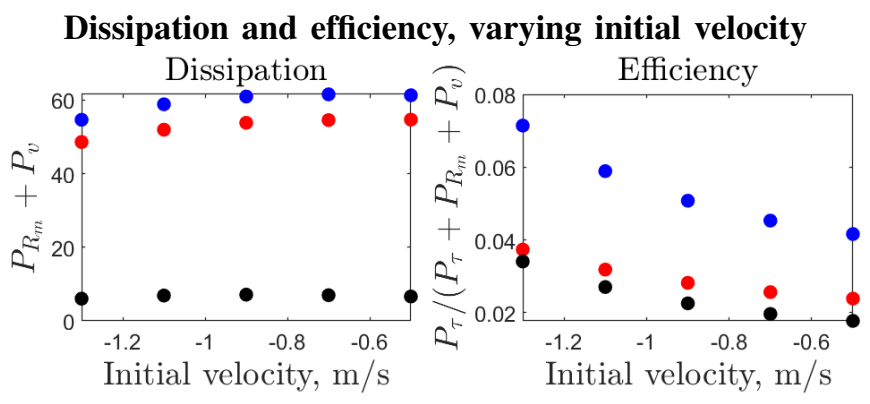

Fig. 4: The simulated dissipation of energy (left; see Eqn 2) over a whole hop for the compression-extension (blue) and active damping (red) controllers is plotted under different initial velocities, with the difference between dissipated energy for the compression-extension and active damping controllers (black). The efficiency of the motors (right; Eqn 2), is plotted for the compression-extension (blue) and active damping (red) controllers, with their difference (black).

\section{Apices and mechanical energy, varying initial velocity}

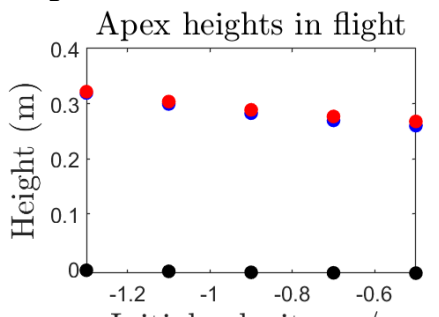

Initial velocity, $\mathrm{m} / \mathrm{s}$

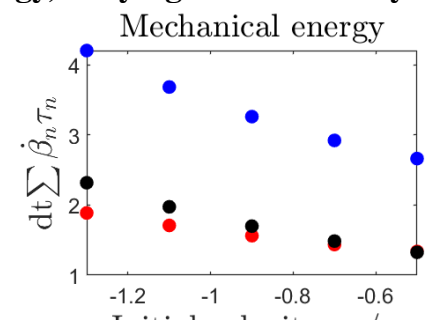

Initial velocity, $\mathrm{m} / \mathrm{s}$
Fig. 5: The apex heights of a simulated robot jumping with the compression-extension controller (blue) and the active damping controller (red; difference in black) are remarkably similar, though the compression-extension controller requires about twice as much mechanical energy to achieve these apex heights.

In the dissipation function, we varied the linear scaling parameter that multiplies the rate of change of the added mass, $b_{g}$. When the cone is fully formed and the dissipation function is quadratic in velocity, this parameter becomes a scalar directly multiplying $\dot{x}_{f}^{2}$.

Using the same initial conditions (leg spring at rest length at start of stance, $-1 \frac{\mathrm{m}}{\mathrm{s}}$ velocity at body and foot) for all simulations comparing ground conditions, the compressionextension controller uses about $20 \%$ more energy than the active damping controller (see Figure 6).

\section{ANALysis}

A. Mechanical energy losses to the ground are incurred both from the ground's dissipation function and from plastic ground deformation

To understand why the compression-extension controller loses more energy than the active damping controller, we must first understand where the energy is lost during a jump on granular media. Examining the total mechanical energy of the system, there are six relevant energies:

1) $L S P$ (leg spring potential): $0.5 \cdot k_{l} \cdot\left(x_{b}-x_{f}-l\right)^{2}$

2) $K B$ (kinetic energy of body): $0.5 \cdot m_{b} \cdot \dot{x}_{b}^{2}$

3) $G B$ (gravitational potential energy of body): $m_{b} \cdot g \cdot x_{b}$ 
Dissipation and efficiency, varying ground parameters

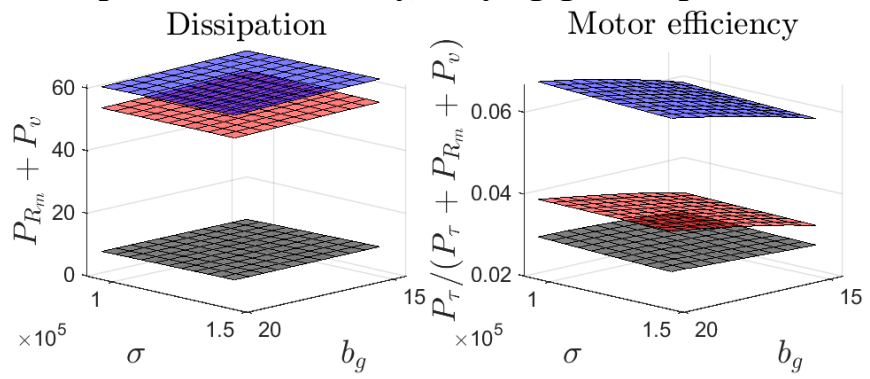

Fig. 6: The simulated dissipated energy (left) over a whole hop for the compression-extension (blue) and active damping (red) controllers is plotted under different ground conditions, with their difference (gray). Here, $\sigma$ indicates the stiffness of the ground, and $b_{g}$ is the linear coefficient on the ground's dissipation function. The efficiency of the motors (right; see Eqn 2) is plotted for the compression-extension (blue) and active damping (red) controllers, with their difference (gray). As when varying initial conditions (Fig. 4 ), we see that even though the compression-extension controller uses its motors more than twice as efficiently as the active damping controller, the compression-extension controller still dissipates almost $20 \%$ more energy.

\section{Apices and mechanical energy, varying ground parameters}

Apex heights in flight Mechanical energy
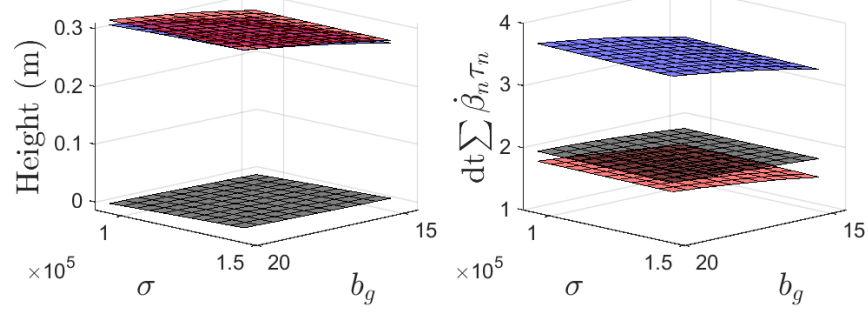

Fig. 7: The apex heights of a simulated jump (left) under the compression-extension (blue) and active damping (red) controllers are almost indistinguishable (difference in gray). However, the mechanical energy output of the motors (right) required to achieve that height is more than twice as large when using the compressionextension (blue) controller as when using the active damping controller (red). Difference in gray.

4) GSP (ground "spring potential"): $\int_{0}^{\left|x_{f}\right|} k_{g}(z) d z$ (recall that $x_{f}$ is negative)

5) $K T$ (kinetic energy of foot): $0.5 \cdot m_{f} \cdot \dot{x}_{f}^{2}$

6) GT (gravitational potential energy of foot): $m_{f} \cdot g \cdot x_{f}$

The total mechanical energy of the body, leg and foot is then

$$
E_{b f}=L S P+K B+G B+K T+G T,
$$

which is the sum of all of these energies except for the ground's "potential" energy, since the ground plastically deforms and any energy transferred to it is immediately lost.

The total energy for one jump in this simulation using the compression-extension and active damping controllers along with all component energies is plotted in Figure 8. The dissipation function of the ground contributes to the initial drop in total energy (black line in plot). The robot's leg, which has a very soft compliance gain during compression, initially deflects more quickly than the ground. This is
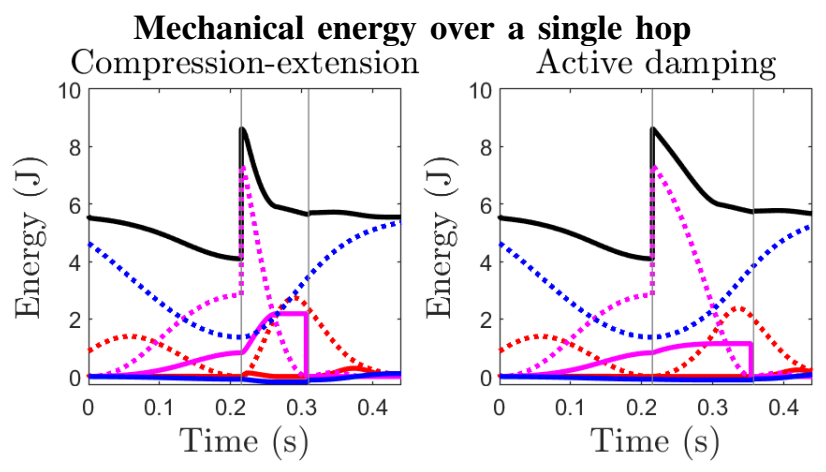

Fig. 8: The total mechanical energy (Eqn 3; black line) during stance is plotted along with the component kinetic (red), spring potential (magenta), and gravitational potential (blue) energies for the body-leg (dotted) and foot-ground (solid) subsystems. The vertical thin gray lines indicate the switch from soft virtual leg spring to stiff at the midpoint of stance and back at toe liftoff.

reflected in the quantity of energy being stored in the robot's virtual leg spring (dotted magenta line), which is larger than the quantity lost to ground compression (solid magenta line). The trajectory of the body during compression is also reflected in the gravitational potential energy of the robot's body mass (blue dotted line).

When the leg stops compressing, $\dot{x}_{b}-\dot{x}_{f} \rightarrow 0$ (first gray line), the compliance gain of its virtual spring suddenly changes to the extension value, injecting a large quantity of spring potential energy (dotted magenta line). Much of this energy is quickly lost to the ground, both through further compression (bump in the solid magenta line) and to the ground's dissipation function. Notice that a small amount of gravitational potential energy is gained by the foot at liftoff (second gray line), because negative gravitational potential energy is lost when the foot mass, which is below 0 height, is suddenly reduced to its nominal value.

Ultimately, the difference in mechanical energy loss between the compression-extension and active damping controllers is very similar, which is reflected in the similar apex heights (Figures 5,7). Notice also that while from the point of view of the physical world the robot's leg looks like a spring-mass damper system, because the leg spring is virtual, the forces that produce this behavior will cost more or less energy from the battery to create depending on the leg's kinematics and the efficiency of its motors. The total mechanical energy is concerned only with the mechanical behavior of virtual springs.

\section{B. Electrical energy cost of locomotion is mitigated by purposefully dissipating energy into the virtual leg damper}

Recall that the ground is modeled as a transiently nonlinear spring with nonlinear dissipation (Sections I-B.1, I-B.2) and no restoring forces. In addition to losing all energy dissipated into the ground, the deeper the robot's foot penetrates into the ground, the higher the robot must jump to achieve the same world-frame height at its apex.

To explore why twice as much mechanical energy is required to jump to the same apex height under the 
compression-extension controller, let us focus for a moment on the mechanical energy in the physical subsystem involving just the foot and ground. We conceptually isolate this subsystem by "zeroing out" the forces from the leg, $F_{b f}$ (Eqn 1), and consider the energy just of the foot-ground subsystem, $E_{f}=K T+G T$, which interacts with the vector field defined over the foot dynamics, $\left\{x_{f}, \dot{x}_{f}\right\}$. The energy used to plastically deform the ground cannot be recovered, so there is only contribution from the kinetic and gravitational potential energy of the foot. The mechanical power loss function of the foot-ground subsystem (Figure 9) is then

$$
\dot{E}_{f}=-d_{g}\left(x_{f}\right) \cdot\left|\dot{x}_{f}{ }^{3}\right|-k_{g}\left(x_{f}\right)\left|\dot{x}_{f}\right| .
$$

Since the dissipation function is quadratic in velocity, the power function is cubic and the ground will dissipate a large amount of energy when the velocity is high. The depth dependence of the first term is due to the formation of the cone of added material, so we expect to see a different slope to the power loss function for high velocities when depths are low. Notice too that this power loss function involves the stiffness function of the ground, $k_{g}\left(x_{f}\right)$, because the ground deforms plastically and does not store energy. Looking at the surface plots in Figure 9, we can see the appropriate dips in the power loss landscape as we vary the depth and the velocity of the foot. Qualitatively, we surmise that if a robot can keep its foot away from these higher-cost parts of its state space in inertial world-frame coordinates, it will lose much less mechanical energy to the ground during stance.

Comparing sample trajectories of the robot's foot through its state space under the compression-extension and active damping controllers (Figure 9), the mechanism of the reported mechanical energy savings for the added damping controller becomes clear. Three sample initial conditions are plotted (black circles). In each initial condition, the initial body and foot velocities are the same, the foot is just touching the ground, and the virtual leg spring is at its rest length. During compression mode, which is the same for the two controllers, the virtual leg spring compresses quickly and the foot's velocity quickly drops as it sinks into the granular media. The foot's velocity goes to zero when the granular media "jams" and returns an equivalent reaction force. When the leg length velocity goes to zero after this point, the virtual leg spring switches to its stiff extension gain, causing a sudden increase in velocity as the forces from the leg spring are now stronger than those exerted by the ground. This enables the foot to continue compressing the ground. The ground jams a second time when the foot's velocity again goes to zero, and the robot's foot begins to lift from the compressed ground, entering ballistic flight.

When the robot leg switches from compression to extension modes, its virtual spring changes stiffness instantaneously and exerts a large force to both push the body up and the foot down further into the ground, as reflected in the large swoop down into the high-depth, high-velocity portion of the foot's state space in the compression-extension trajectory plots in Figure 9. The compression-extension controller injects a large amount of energy into the foot-ground
Sample trajectories in the foot's state space under compression-extension and active damping controllers
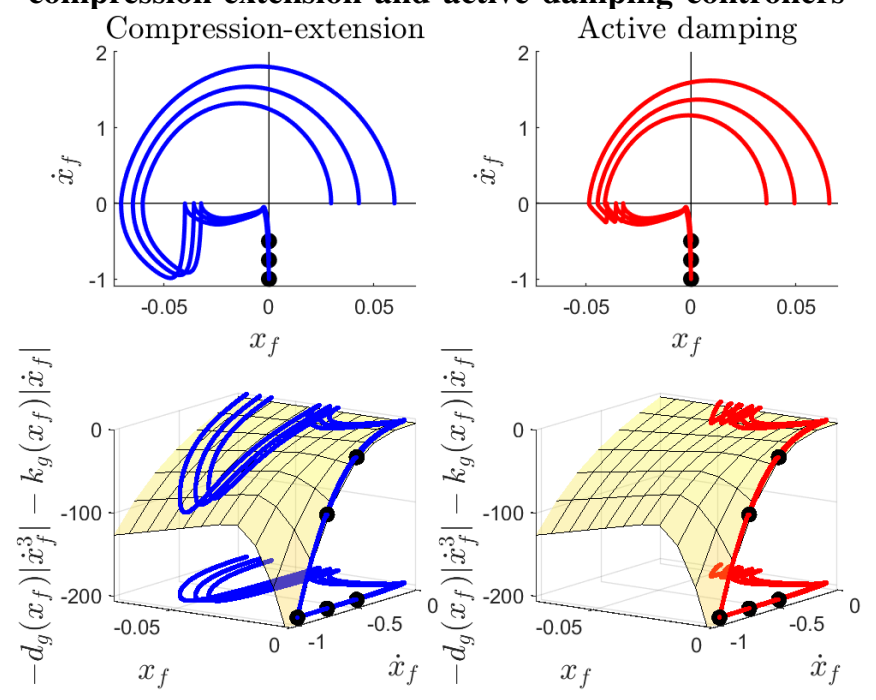

Fig. 9: The power function of the ground (yellow surface; Eqn 4) shows large losses during high-velocity intrusions because of the dissipation function of the ground and during high-depth intrusions because of its plastic deformation. The steep dip in the low-depth portion of the power loss function is due to the formation of the cone of material accelerated with the foot. A comparison of sample trajectories from three initial conditions (black circles) through the foot's state space in the inertial world frame using the compressionextension (blue) and the active damping (red) controllers shows decreased losses for the active damping controller during extension.

system when the velocity of the leg length goes to zero, but loses most of this energy immediately to the ground. In contrast, the active damping controller punishes negative foot velocities, pushing the state of the foot back towards zero velocity. Since the foot's velocity is negative for a smaller amount of time, it does not penetrate as far into the ground and does so less quickly, and thus loses less mechanical energy.

The energy being lost by the foot-ground subsystem during stance is largely energy that is injected into it by the leg spring force $F_{b f}$ (Eqn 1). Kinetic energy losses to mechanical dissipation in the leg spring may be significant when its damping coefficient is high. However, recall that the spring and damper in the leg are both virtual, and all forces exerted by a virtual leg spring come at a cost from the battery. This includes leg spring potential energy storage, which in a mechanical system is free. Virtual damping forces acting in opposition to virtual spring forces that reduce the torque requested from the motors will therefore use less energy from the battery.

While the added damping force in the virtual leg spring does potentially come at a cost in kinetic energy, the investment is rewarded immediately in two ways: First, the ground compresses less, so that the robot does not need to jump as high from the point underneath the surface of the granular media at which it enters flight mode in order to achieve the same apex height in an inertial frame; and second, the power function of the ground has a lower magnitude when 
the foot's depth and velocity are lower, so less mechanical energy is dissipated to the ground. In fact, the apex heights of the compression-extension and active damping controllers are comparable, and in some cases higher when active damping is used (see Figures 5, 7), even though the compression-extension controller requires more than twice as much mechanical energy to achieve those heights across different initial conditions and ground parameters.

\section{Conclusions}

We suggest using computer simulations of a monoped jumping on granular media that well studied and robust controllers with good extensibility like the Raibert compressionextension controller can be composed with simple reactive controllers like the active damping controller introduced here to produce significant energy cost reductions for locomotion on plastically deforming ground with high dissipation, without needing to model the ground online. The mitigation of mechanical energy loss to the ground through purposeful energy dissipation by the leg spring also provides context for previous results on robot jumping in granular media.

Because the active damping controller reduces the energy loss to dissipation and requires half the mechanical power of the compression-extension spring to jump to the same height for a range of ground and initial conditions, and because the energy savings occur after the cone of added material is already fully formed, it is possible that the results may extend to locomotion on other types of fragile ground. Of most relevant to the present research application is performance on inclined granular media. On inclines, although the gravity vector is not normal to the surface of the ground, the media exhibit similar qualitative bulk behavior [22], suggesting that energetic costs may also be reduced using the active damping controller. We plan to follow up this work with experimental studies using a sandproofed desertready Minitaur to demonstrate the efficacy of this method in aiding desert research.

\section{ACKNOWLEDGMENT}

This work was supported by NSF INSPIRE grant 1514882 and in part by NSF NRI-2.0 grant 1734355 . We also gratefully acknowledge Drs. Feifei Qian and Chen Li for discussions about the granular media force laws referenced for this work; Jeffrey Duperret and Wei-Hsi Chen for discussions about motor and battery models; Ghost Robotics for technical support; and Diedra Krieger for administrative support.

\section{REFERENCES}

[1] K. Galloway, G. C. Haynes, B. D. Ilhan, A. Johnson, R. Knopf, G. Lynch, B. Plotnick, M. White, and D. E. Koditschek, "X-RHex: A highly mobile hexapedal robot for sensorimotor tasks," 2010.

[2] S. F. Roberts, J. M. Duperret, X. Li, H. Wang, and D. E. Koditschek, "Desert RHex technical report: Tengger desert trip," 2014.

[3] S. F. Roberts, J. M. Duperret, A. M. Johnson, S. van Pelt, T. Zobeck, N. Lancaster, and D. E. Koditschek, "Desert RHex technical report: Jornada and white sands trip," 2014.

[4] G. Kenneally, A. De, and D. E. Koditschek, "Design principles for a family of direct-drive legged robots," IEEE Robotics and Automation Letters, vol. 1, no. 2, pp. 900-907, 2016.
[5] F. Qian, D. Jerolmack, N. Lancaster, G. Nikolich, P. Reverdy, S. F. Roberts, T. Shipley, R. S. Van Pelt, T. M. Zobeck, and D. E. Koditschek, "Ground robotic measurement of aeolian processes," Aeolian Research, vol. 27, pp. 1-11, 2017.

[6] A. De and D. E. Koditschek, "Vertical hopper compositions for preflexive and feedback-stabilized quadrupedal bounding, pacing, pronking, and trotting," The International Journal of Robotics Research, vol. 37, no. 7 , p. 743778, 2018.

[7] G. Kenneally, W.-H. Chen, and D. E. Koditschek, "Actuator transparency and the energetic cost of proprioception," ISER, 2018 (accepted).

[8] B. D. Ilhan, A. M. Johnson, and D. E. Koditschek, "Autonomous legged hill ascent," Journal of Field Robotics, vol. 35, p. 802832, Aug 2018.

[9] J. J. Aguilar and D. I. Goldman, "Robophysical study of jumping dynamics on granular media," Nature Physics, vol. 12, no. 3, p. 278, 2016.

[10] C. Li, T. Zhang, and D. I. Goldman, "A terradynamics of legged locomotion on granular media," Science, vol. 339, no. 6126, pp. 1408$1412,2013$.

[11] K. Kamrin, "A hierarchy of granular continuum models: Why flowing grains are both simple and complex," in EPJ Web of Conferences, vol. 140, p. 01007, EDP Sciences, 2017.

[12] J. Slonaker, D. C. Motley, Q. Zhang, S. Townsend, C. Senatore, K. Iagnemma, and K. Kamrin, "General scaling relations for locomotion in granular media," Physical Review E, vol. 95, no. 5, p. 052901, 2017.

[13] A. H. Chang, C. M. Hubicki, J. J. Aguilar, D. I. Goldman, A. D. Ames, and P. A. Vela, "Learning to jump in granular media: Unifying optimal control synthesis with Gaussian process-based regression," in Robotics and Automation (ICRA), 2017 IEEE International Conference on, pp. 2154-2160, IEEE, 2017.

[14] C. M. Hubicki, J. J. Aguilar, D. I. Goldman, and A. D. Ames, "Tractable terrain-aware motion planning on granular media: an impulsive jumping study," in Intelligent Robots and Systems (IROS), 2016 IEEE/RSJ International Conference on, pp. 3887-3892, IEEE, 2016.

[15] F. Qian, T. Zhang, W. Korff, P. B. Umbanhowar, R. J. Full, and D. I. Goldman, "Principles of appendage design in robots and animals determining terradynamic performance on flowable ground," Bioinspiration \& biomimetics, vol. 10, no. 5, p. 056014, 2015.

[16] W. Kang, Y. Feng, C. Liu, and R. Blumenfeld, "Archimedes law explains penetration of solids into granular media," Nature coтmиnications, vol. 9, no. 1, p. 1101, 2018.

[17] C. S. Bester and R. P. Behringer, "Collisional model of energy dissipation in three-dimensional granular impact," Physical Review E, vol. 95 , no. 3, p. 032906, 2017.

[18] A. H. Clark and R. P. Behringer, "Granular impact model as an energydepth relation," EPL (Europhysics Letters), vol. 101, no. 6, p. 64001, 2013.

[19] M. H. Raibert, Legged robots that balance. MIT press, 1986.

[20] A. De and D. E. Koditschek, "Parallel composition of templates for tail-energized planar hopping," in Robotics and Automation (ICRA), 2015 IEEE International Conference on, pp. 4562-4569, IEEE, 2015.

[21] A. De and D. E. Koditschek, "The Penn Jerboa: A platform for exploring parallel composition of templates," arXiv preprint arXiv:1502.05347, 2015.

[22] Y. Forterre and O. Pouliquen, "Flows of dense granular media," Апnи. Rev. Fluid Mech., vol. 40, pp. 1-24, 2008. 